\title{
Biomimetic Oral Appliance Therapy in Adults with Mild to Moderate Obstructive Sleep Apnea Using Combined Maxillo- Mandibular Correction
}

\author{
G Dave Singh ${ }^{1 *}$ and Samuel E Cress ${ }^{2}$
}

${ }^{1}$ Vivos BioTechnologies, Beaverton, USA

${ }^{2}$ Craniofacial and Dental Sleep Medicine, Sugar Land, TX, USA

${ }^{*}$ Corresponding author: Prof. G Dave Singh, Vivos BioTechnologies, 1915 NW Amberglen Parkway, Suite 400, Beaverton, OR 97006, USA, Tel: +1-971-302-2233, E-mail: drsingh@drdavesingh.com

\begin{abstract}
Introduction: Although mandibular advancement devices (MADs) are utilized for the management of mild to moderate OSA, there are concerns about temporo-mandibular joint (TMJ) issues. Biomimetic oral appliance therapy (BOAT) differs from MADs as it aims to achieve midfacial redevelopment in combination with mandibular repositioning. In this study, we tested the hypothesis that mild to moderate cases of OSA can be addressed with combined maxillo-mandibular correction using BOAT.
\end{abstract}

Methods: Nineteen adults diagnosed with mild to moderate OSA, following an overnight home sleep test (HST) that had been interpreted by a physician, participated in this study. Each subject was treated by a dentist with advanced training in dental sleep medicine. At each monthly followup, the devices were adjusted to optimize their efficacy. The mean apnea-hypopnea index ( $\mathrm{AHI})$, respiratory disturbance index (RDI) and oxygen desaturation index (ODI) of the study sample was calculated prior to and after BOAT. The findings were subjected to statistical tests.

Results: Prior to treatment the mean $\mathrm{AHI}$ of the study subjects was $12.8 \mathrm{hr}^{-1} \pm 5$; the mean RDI was $18.6 \mathrm{hr}^{-1} \pm$ 8.2 , and the ODI was $6.3 \% \pm 3.5$. Follow-up HSTs after approximately 9 mos. indicated the mean $\mathrm{AHI}$ decreased significantly to $6.2 \mathrm{hr}^{-1} \pm 2.9$ after BOAT ( $p<0.001$ ), which represents a fall in the mean $\mathrm{AHI}$ by $51.5 \%$ for the study sample. The mean RDI fell to $12.3 \mathrm{hr}^{-1} \pm 6.9(\mathrm{p}<0.001)$, and the ODI was improved to $2.6 \% \pm 1.7(p<0.001)$.

Conclusions: This study suggests that combined maxillomandibular correction may be a useful method of managing mild to moderate cases of OSA in adults, and represents an alternative to MADs and continuous positive airway pressure therapy. However, further studies are needed to determine the additional advantages of combined maxillomandibular correction.

\author{
Keywords \\ Biomimetic, Oral appliance therapy, Palatal expansion, Man- \\ dibular advancement
}

\begin{abstract}
Abbreviations
MAD: Mandibular Advancement Device; OSA: Obstructive Sleep Apnea; TMJ: Temporo-Mandibular Joint; BOAT: Biomimetic Oral Appliance Therapy; HST: Home Sleep Test; AHI: Apnea-Hypopnea Index; RDI: Respiratory Disturbance Index; ODI: Oxygen Desaturation Index; CPAP: Continuous Positive Airway Pressure
\end{abstract}

\section{Introduction}

Obstructive sleep apnea (OSA) is a common sleep disorder with serious medical implications. Although several therapeutic options are available for this condition, patient compliance may result in inadequate treatment. For example, continuous positive airway pressure (CPAP) therapy is not well-tolerated due to unwanted side effects, and patient compliance can be as low as 50\% [1]. Thus, other treatment options for OSA include: weight loss, positional therapy, oral appliance therapy, hypoglossal nerve stimulation, and surgery. Indeed, in a pilot study, the surgical removal of extensive, bilateral torus mandibularis decreased the apnea-hypopnea index (AHI) by $15 \%$ in a 47 -year-old male with moderate OSA [2]. However, instead of using various treatments alone, clinical outcomes might be improved by combining different treatment modalities [2]. For instance, surgical treatment is used in cases of OSA where CPAP or mandibular advancement device (MAD) failures may have occurred. In this respect, the role of surgery is to facilitate long-term device tolerance or use [3]. For example Başal, et

Citation: Singh GD, Cress SE (2017) Biomimetic Oral Appliance Therapy in Adults with Mild to Moderate Obstructive Sleep Apnea Using Combined Maxillo-Mandibular Correction. J Sleep

C Disord Manag 3:014

Received: December 23, 2016: Accepted: March 14, 2017: Published: March 17, 2017

International Library Copyright: (c) 2017 Singh GD, et al. This is an open-access article distributed under the terms of the Creative Commons Attribution License, which permits unrestricted use, distribution, and reproduction in any medium, provided the original author and source are credited. 
al. described a severe case of OSA that was treated with combined surgical methods [4]. Post-operatively, the AHI was radically-reduced while using CPAP and, post-surgically, the CPAP intolerance was eliminated. Thus, a combination of surgical methods might increase CPAP compliance.

Indeed, other treatments such as weight loss, nasal surgery and positional devices may be combined with surgical procedures of the upper airway. Azbay, et al. studied multilevel upper airway surgery on CPAP tolerance [5].

Procedures such as septoplasty and uvulo-pharyngo-palatoplasty (with or without modified tongue base suspension) were deployed. Post-operatively, about $50 \%$ of the study sample reported that their symptoms were resolved, and about $50 \%$ tolerated CPAP without problems. On the other hand, Hui, et al. investigated the therapeutic effects of a MAD combined with either nasal or pharyngeal surgery [6]. The AHI of the combined surgical group was significantly lower post-operatively, and the authors concluded that surgical enlargement of the nasal and pharyngeal airways combined with a MAD is an effective treatment in patients with severe OSA. Similarly, Wang, et al. evaluated the effect of CPAP combined with a MAD in the treatment of severe OSA [7]. After 3 months of treatment, the AHI in the $\mathrm{CPAP}$-combined with MAD group and the CPAP-alone group were significantly lower than that in the MAD-group alone; but there was no difference in AHI between the CPAP-combined with MAD group and the CPAP-alone group. However, the oxygen desaturation index (ODI) in the CPAP-combined with MAD group was significantly lower than that in the CPAP-alone group. Therefore, the authors concluded that CPAP combined with a MAD was better than CPAP or MADs alone in the treatment of severe OSA.

While MADs have long been successfully deployed for the management of OSA, either in isolation or in combination, there is some concern about temporo-mandibular joint (TMJ) symptoms, changes in the occlusion and unwanted tooth movements [8]. Biomimetic oral appliance therapy (BOAT) differs from conventional MADs as it aims to correct the nasal airway [9] through midfacial redevelopment [10] in combination with mandibular repositioning, which aims to improve the oropharyngeal airway in adults [11]. But, there are very few studies in the literature that have investigated a combination of midfacial development in conjunction with mandibular repositioning in the treatment of OSA. Therefore, in this study we tested the hypothesis that mild to moderate cases of OSA can be addressed using a combined maxillo-mandibular protocol in adults.

\section{Methods and Sample}

After obtaining informed consent, we included 19 consecutive adults that had been diagnosed with mild to moderate OSA following an overnight home sleep test (HST). The rights of the subjects were protected by following the Declaration of Helsinki. Inclusion criteria were: subjects aged $>21 \mathrm{yrs}$; diagnosed without severe

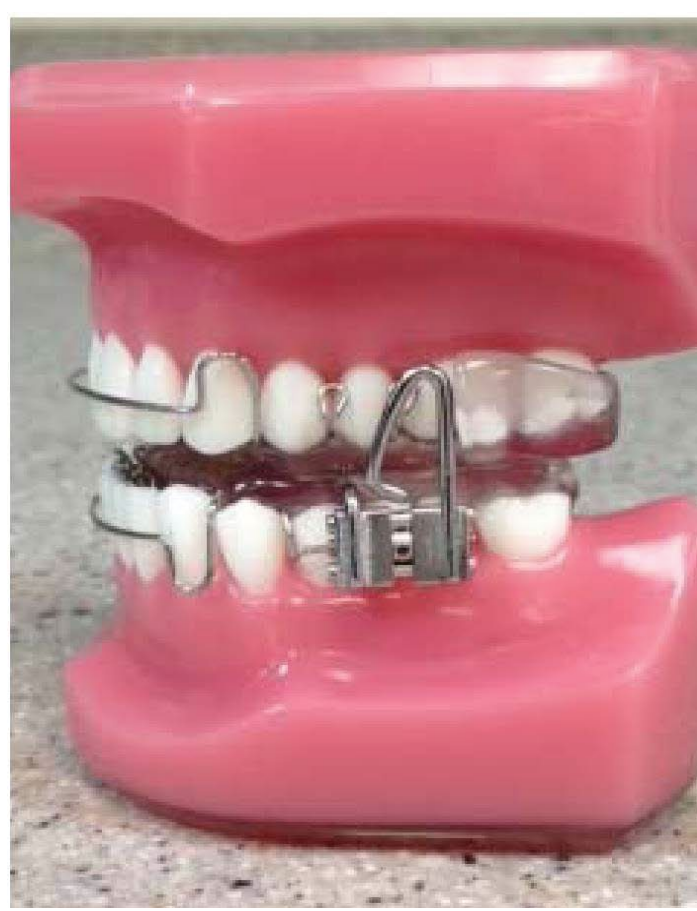

Figure 1: The biomimetic oral appliance (mandibular-Repositioning Nighttime Appliance: mRNA appliance ${ }^{\circledR}$ ) used in this study. The mRNA appliance ${ }^{\circledR}$ is FDA-cleared as a Class II medical device for mild to moderate cases of obstructive sleep apnea. Note that the lower component has a screw-fin mechanism to titrate the mandibular repositioning in $1 \mathrm{~mm}$ increments.

OSA following an overnight sleep study that had been interpreted by a physician; good oral appliance compliance; no history of hospitalization for craniofacial trauma or surgery; no congenital craniofacial anomalies, and dentate upper and lower arches. The exclusion criteria included: age $<21$ yrs; lack of oral appliance compliance; active periodontal disease; excessive tooth loss; poor oral hygiene, and systemic bisphosphonate therapy.

Following a confirmed diagnosis of mild to moderate OSA by a medical physician, careful history-taking and craniofacial examination was undertaken for each subject by a dentist (SEC) with advanced training in dental sleep medicine, and each subject was treated using BOAT. BOAT differs from the conventional MADs as it aims to remodel the upper airway through combined maxillo-mandibular correction with midfacial development and mandibular repositioning. Therefore, a bite registration was obtained in the upright-sitting position with the jaw posture corrected in the antero-posterior and vertical axes specific for each subject, using a sibilant phoneme registration protocol or 'phonetic bite' [12]. Upper and lower polyvinyl siloxane impressions were also obtained. The upper model was then mounted on an articulator and the lower model was mounted relative to the upper model, using the bite registration captured. Blinded to one investigator (GDS), a biomimetic oral appliance with mandibular repositioning capabilities (mandibular-Repositioning Nighttime Appliance: mRNA appliance ${ }^{\circledR}$; Figure 1) was prescribed for the study population. The mRNA appliance ${ }^{\circledR}$ is FDA-cleared 
as a Class II medical device for mild to moderate cases of OSA. The BOAT protocol has previously been deployed to address maxillo-mandibular and upper airway deficiencies in both children and adults [13-18]. All appliances used in this study had: a labial bow; 6 anterior 3-D axial springs ${ }^{\mathrm{TM}}$; a midline screw; bilateral occlusal coverage; retentive slots (Figure 1); a beaded, pharyngeal stent (Figure 2), and a nighttime mandibular repositioning component (Figure 3) with a screw-fin mechanism to titrate the mandibular advancement in $1 \mathrm{~mm}$ increments. All subjects were instructed to wear the appliances during the evening and at nighttime (for approx. 12-16hrs in total), but not during the day time and not while eating, partly in line with the circadian

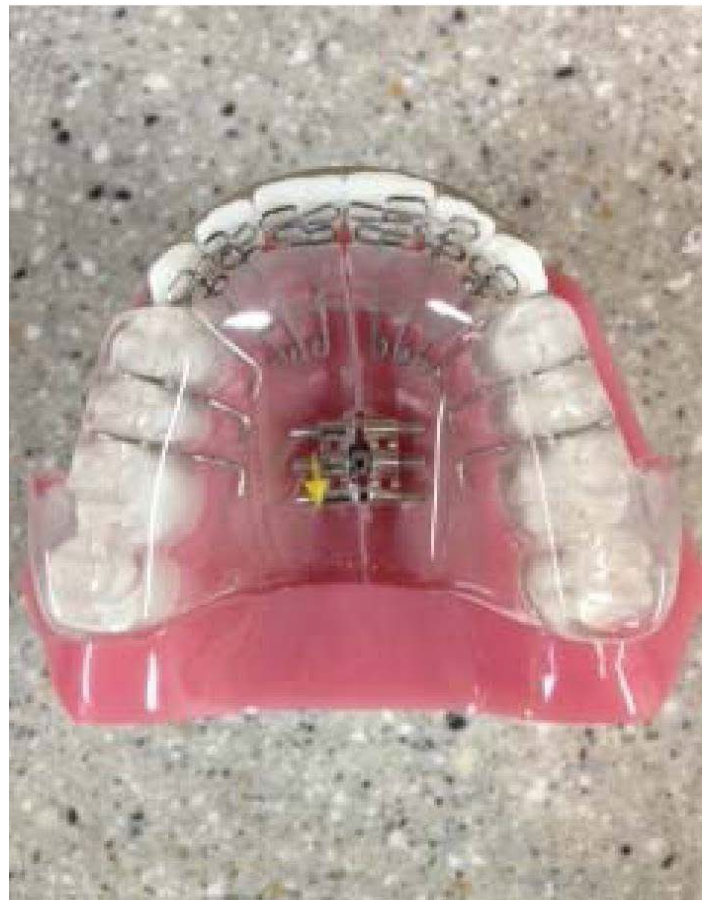

Figure 2: The upper component of the biomimetic oral appliance used in this study with: 6 anterior 3-D axial springs $^{\mathrm{TM}}$, a midline screw, bilateral occlusal coverage, retentive slots, and a labial bow. rhythm of tooth eruption [19], although this only occurs in children. Proffit [20] notes that an appliance needs to be worn for at least $8 \mathrm{hrs}$ in the mouth to have a clinical effect. Written and verbal instructions were given to all subjects.

BOAT requires professional adjustments approximately every 4 weeks, and all subjects reported for review each month. At each monthly follow-up, examination for the progress of craniofacial correction was recorded. Adjustments of the devices were performed to optimize their efficacy. The subjects were encouraged to maintain their treatment protocol as outlined at the beginning of treatment. Specifically, the tension/retention of the device was maintained by advancing the expansion screw in the upper component (Figure 1) by approx. $0.25 \mathrm{~mm}$ weekly. The labial bow was adjusted so that it did not contact the labial surfaces of the anterior teeth. Gradually, the fitting surface acrylic/posterior border of the device was reduced to permit the hard palate to remodel inferiorly. The occlusal acrylic was also selectively equilibrated and gradually removed, to

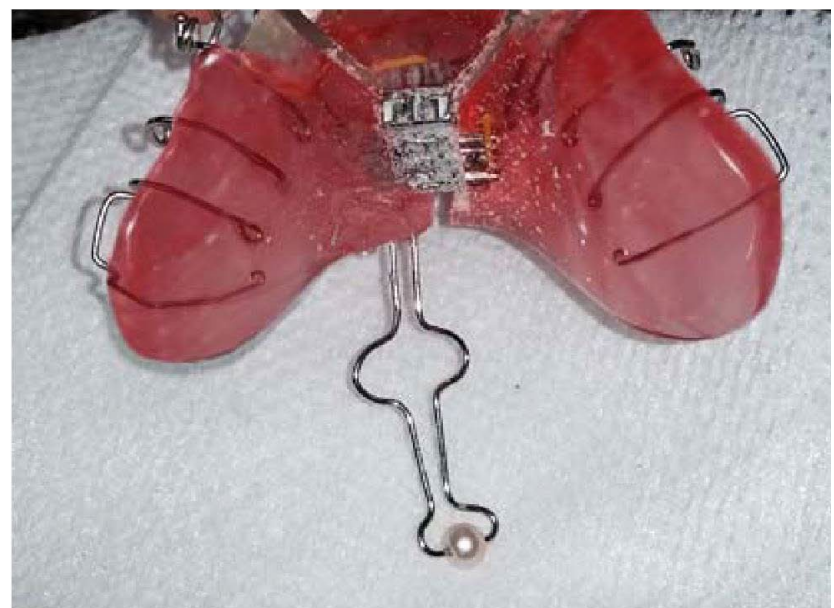

Figure 3: The upper component of the biomimetic oral appliance used in this study showing the beaded, pharyngeal stent, which was adjusted to prevent the tongue and uvula from obstructing the upper airway.

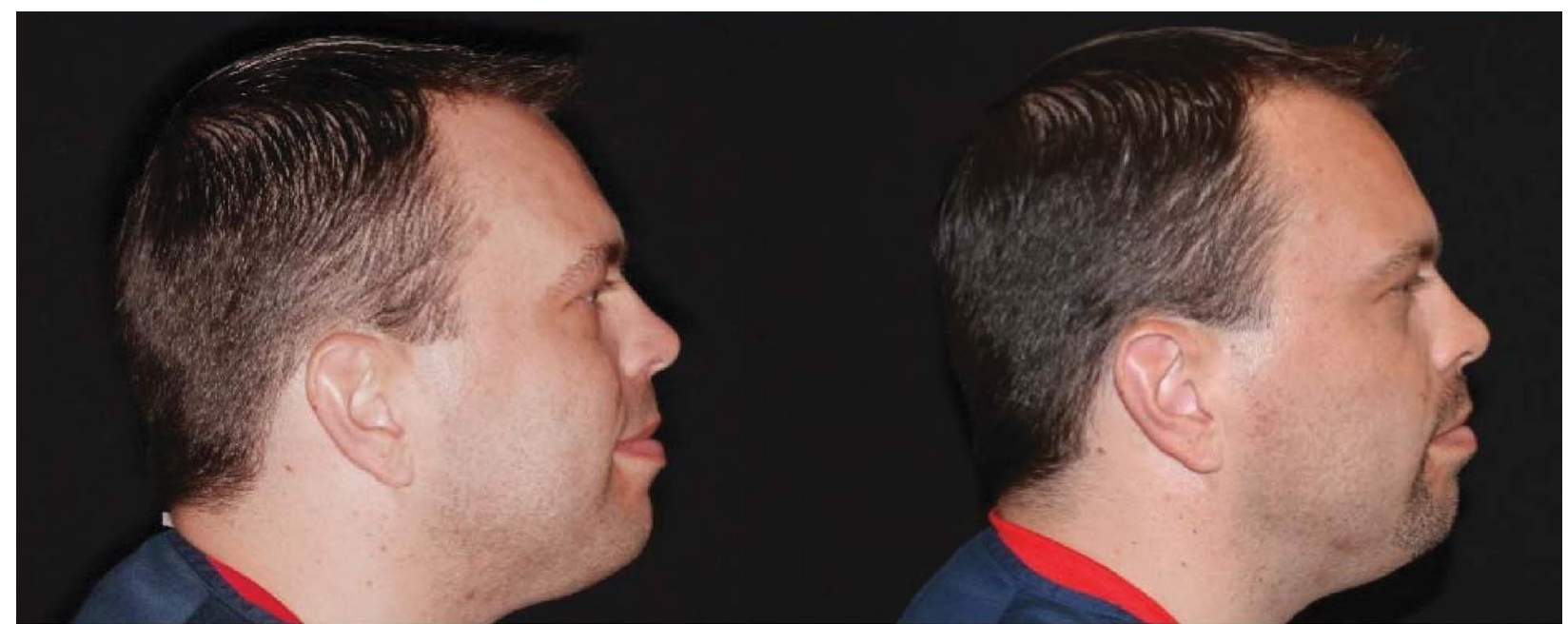

Figure 4: The changes in facial profile and head posture in a subject that participated in this study. The figure on the left shows the subject prior to treatment. The figure on the right shows the same subject after approx. 9 mths using the combined maxillo-mandibular protocol. 
permit the lower teeth to track the changes in the occlusion. The beaded, pharyngeal stent (Figure 2) was adjusted to prevent the tongue and uvula from obstructing the upper airway. Finally, the 3D axial springs (Figure 1) were adjusted so that only gentle pressures were transmitted to the teeth and surrounding tissues. The functionality of the devices was checked with the subject activating a mild force on biting. Advancement of the mandible and development of the lower arch was implemented using the separate lower component (Figure 3). To permit arch re-coordination, the midline expansion screw of the lower component was activated. In addition, the screw-fin mechanism (Figure 3) was adjusted in $1 \mathrm{~mm}$ increments to reposition the mandible in the position required to maintain an adequate upper airway at nighttime for sleep. The post-treatment HSTs were done approx. 9mos later and were interpreted by a medical physician. The mean AHI, RDI and ODI of the study sample were calculated prior to and after BOAT, and the findings were subjected to statistical analysis, using paired t-tests.

\section{Results}

Figure 4 is an example that shows the changes in facial profile and head posture in a subject that participated in this study. Figure 5 is an example that shows study models indicating the intra-oral, maxillo-mandibular changes in a different subject who underwent the same procedure. Figure 6 shows the upper airway changes in another case treated using the same combined maxillomandibular protocol.

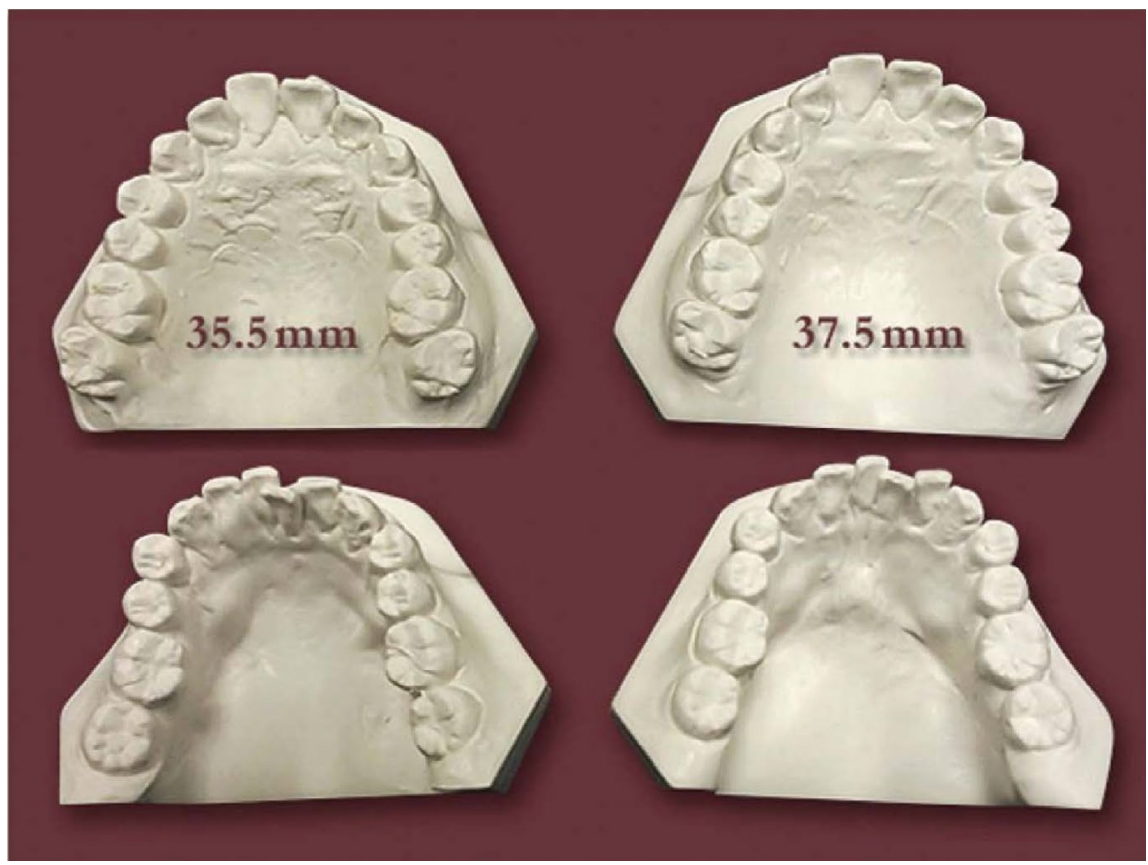

Figure 5: Study models showing the intra-oral changes in a subject who underwent the combined maxillo-mandibular protocol. Note that the minimum inter-molar bone width of the maxilla has increased from $35.5 \mathrm{~mm}$ to $37.5 \mathrm{~mm}$ and the arch has become more symmetric and less crowded. Similarly, the mandibular arch shows improved alignment and width simultaneously.

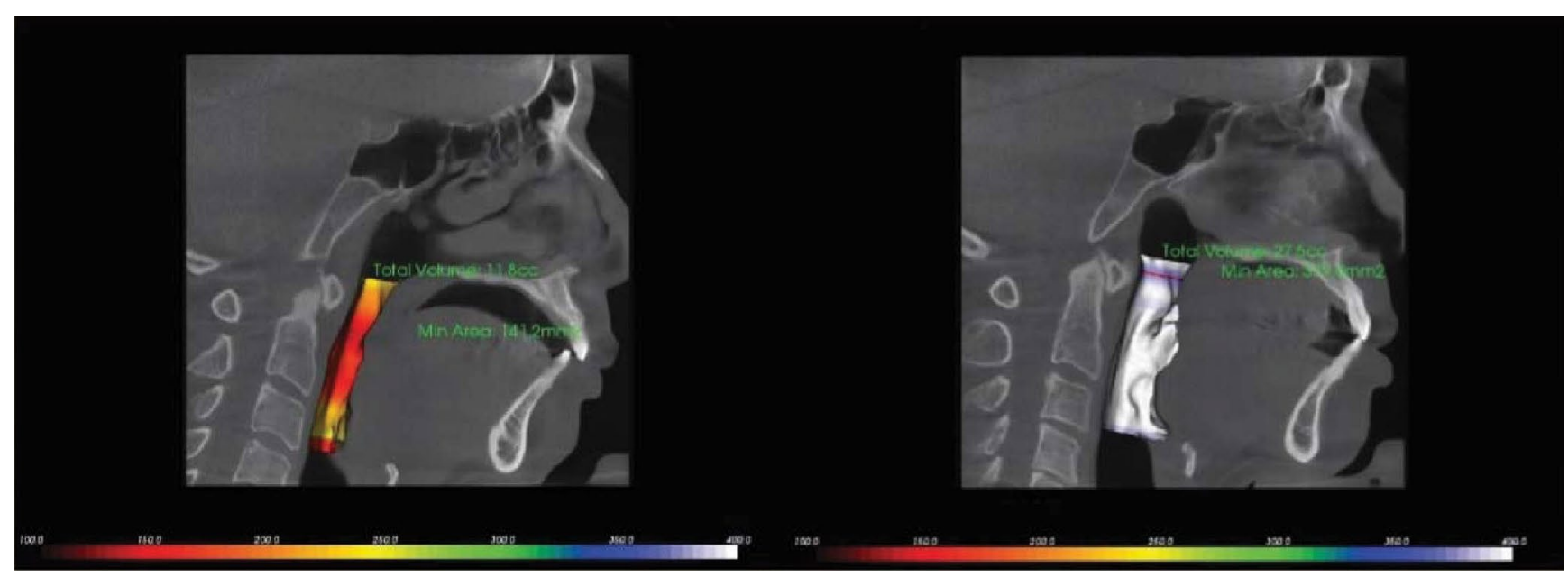

Figure 6: Mid-sagittal slices from a 3D cone-beam computerize axial scan, showing the upper airway changes using the same combined maxillo-mandibular protocol. Note that the upper volume has increased from $11.8 \mathrm{~cm}^{3}$ pre-treatment to $27.5 \mathrm{~cm}^{3}$ post-treatment, while the minimum cross-sectional area has increased from $141.2 \mathrm{~cm}^{2}$ to $379.8 \mathrm{~cm}^{2}$ post-treatment, using the combined maxillo-mandibular protocol. 
Table 1: Summary of sleep parameters assessed in this study.

\begin{tabular}{|c|c|c|c|c|c|c|c|}
\hline \multirow{2}{*}{\begin{tabular}{|l|} 
Subject \\
1 \\
\end{tabular}} & \multicolumn{2}{|c|}{$\begin{array}{l}\text { Pre-treatment } \\
\text { AHI }\end{array}$} & \multirow{2}{*}{$\begin{array}{l}\text { Post-treatment } \\
\text { AHI } \\
\\
\end{array}$} & $\begin{array}{l}\text { Pre-treatment } \\
\text { RDI }\end{array}$ & $\begin{array}{l}\text { Post-treatment } \\
\text { RDI }\end{array}$ & $\begin{array}{l}\text { Pre-treatment } \\
\text { ODI }\end{array}$ & $\begin{array}{l}\text { Post-treatment } \\
\text { ODI }\end{array}$ \\
\hline & & 14.5 & & 15.5 & 12 & 7.4 & 1.2 \\
\hline 2 & & 11.9 & 6 & 13.6 & 7 & 5.5 & 3.9 \\
\hline 3 & & 22.6 & 13.4 & 24.4 & 15.2 & 10.1 & 5.1 \\
\hline 4 & & 2.4 & 0.9 & 9.8 & 7.5 & 1.1 & 0.4 \\
\hline 5 & & 11.3 & 3.6 & 28 & 26.8 & 3.7 & 1 \\
\hline 6 & & 15.4 & 6.8 & 33.2 & 17.5 & 7.9 & 2.4 \\
\hline 7 & & 6.5 & 4.6 & 11.7 & 7.6 & 1.9 & 1.4 \\
\hline 8 & & 15.2 & 10.6 & 21.7 & 16.1 & 5.9 & 5.9 \\
\hline 9 & & 15.6 & 7.6 & 21 & 12.3 & 5.9 & 3 \\
\hline 10 & & 13.7 & 8.9 & 23.2 & 16 & 6.7 & 4.8 \\
\hline 11 & & 11.6 & 5.5 & 6.5 & 2 & 9.2 & 4.4 \\
\hline 12 & & 7.1 & 4 & 7.8 & 4.9 & 3.7 & 1.8 \\
\hline 13 & & 21.4 & 8.3 & 23.4 & 11.4 & 12.6 & 1.7 \\
\hline 14 & & 9.7 & 2.5 & 12.3 & 5 & 3.2 & 0.3 \\
\hline 15 & & 18.8 & 4 & 26.7 & 11.2 & 14.2 & 1 \\
\hline 16 & & 12 & 8 & 15 & 9.5 & 3.8 & 2.4 \\
\hline 17 & & 10.4 & 7.4 & 11.6 & 12.9 & 4.9 & 3.6 \\
\hline 18 & & 15.4 & 5.5 & 33.2 & 28.7 & 7.9 & 1.2 \\
\hline \multirow[t]{3}{*}{19} & & 7.8 & 4.9 & 13.9 & 9.3 & 3.4 & 3.6 \\
\hline & Mean & 12.8 & 6.2 & 18.6 & 12.3 & 6.3 & 2.6 \\
\hline & std & 5 & 2.9 & 8.2 & 6.9 & 3.5 & 1.7 \\
\hline
\end{tabular}

$\mathrm{AHI}=$ Apnea Hypopnea Index; RDI = Respiratory Disturbance Index; ODI = Oxygen Desaturation Index.

Prior to treatment the mean AHI of the study subjects was $12.8 \mathrm{hr}^{-1} \pm 5$; the mean RDI was $18.6 \mathrm{hr}^{-1} \pm 8.2$, and the ODI was $6.3 \% \pm 3.5$. The follow-up HSTs done after approx. 9mos. showed that the AHI decreased significantly $(\mathrm{p}<0.001)$ to a mean value of $6.2 \mathrm{hr}^{-1} \pm 2.9$ after BOAT, which represents a fall in the mean AHI by $51.5 \%$ for the study sample. The mean RDI fell to $12.3 \mathrm{hr}^{-1}$ $\pm 6.9(\mathrm{p}<0.001)$, and the ODI was improved to $2.6 \% \pm$ 1.7 ( $\mathrm{p}<0.001)$. Table 1 summarizes the overall findings. Therefore, it appears that upper airway parameters can be improved in adults with mild to moderate OSA by targeting midfacial correction in combination with mandibular advancement using BOAT.

\section{Discussion}

Obstructive sleep apnea (OSA) is associated with increased cardiovascular diseases, such as high blood pressure and heart failure, as well as metabolic morbidities. In addition, the risk of OSA-related motor vehicle collisions is increased. Treatment of OSA includes changes in diet and lifestyle, CPAP, various oral devices, and upper airway surgical treatments [21]. In this study, we investigated the novel concept that combined maxillo-mandibular oral appliance therapy may be a useful method of managing mild to moderate cases of OSA in adults. This procedure represents an alternative to MAD therapy, where there may be TMJ concerns, or to CPAP therapy, where there might be compliance issues, although we did not directly compare MADs with combined maxillo-mandibular correction in this study. In fact, there are very few studies on simultaneous maxillo-mandibular correction. Dieltjens, et al. used a combination of positional therapy with a MAD for OSA [22].
While, both MAD and positional therapy were individually effective in reducing the AHI, the combination further reduced the overall AHI compared with either method alone. Therefore, there may be advantages to combined therapies in contrast to using one intervention alone, but there may be confounding factors.

Obesity is a major risk factor in the development of OSA. Indeed, Chirinos, et al. assigned patients with obesity and moderate-to-severe OSA to receive treatment with either CPAP, weight-loss intervention, or a combination of the two [23]. The combined interventions showed reductions in insulin resistance and serum triglyceride levels, but, while these changes were observed in the weight-loss group they were not noted in the group receiving CPAP alone. However, weight loss has a variable effect on the severity of OSA, and differences in the effect of weight loss on OSA may be due to underlying craniofacial characteristics. In this respect, Naughton, et al. studied mandibular length in association with changes in AHI following weight loss [24]. Their analysis indicated that the change in AHI correlated with a shorter mandibular body length. Thus, weight loss for severe OSA in obese patients may be useful in those with a shorter mandibular body length. As we did not measure mandibular lengths in this present study, additional studies are needed using $3 \mathrm{D}$ imaging techniques to quantify the craniofacial changes induced with combined BOAT. Nevertheless, the mandibular repositioning device we deployed in combination with midfacial redevelopment decreased the mean AHI by over $50 \%$ and to $<10 \mathrm{hr}^{-1}$ in a sample of non-obese, adult patients, which represents a successful outcome in a medical context. 
Zhang, et al. explored soft palatal surgery combined with uvulo-pharyngoplasty and inferior turbinate radiofrequency for the treatment of OSA [25]. The AHI in their study sample was $16 \mathrm{hr}^{-1}$ at $6 \mathrm{mos}$ but relapsed to about $21 \mathrm{hr}^{-1}$ at $12 \mathrm{mos}$ post-operatively. Therefore, not all combination protocols yield enhanced results. In contrast, following a study that demonstrated beneficial changes after surgical correction of skeletal Class III cases [26], double jaw surgery is currently considered advantageous because it produces an increase in the pharyngeal airway dimensions. In this present study, non-surgical, double jaw correction also appears to enhance the upper airway (Figure 6) and this was confirmed since the mean ODI improved to $2.6 \%$ in the sample we tested after treatment. In addition, it is likely that the sleep architecture may have also improved since the mean RDI fell to $12.3 \mathrm{hr}^{-1}$ in our present study. Therefore, although long-term follow up using a larger sample size is needed to make more definitive conclusions on these initial findings, this present study suggests that combined, maxillo-mandibular, biomimetic oral appliance therapy with midfacial development and mandibular advancement, may be a useful method of managing non-severe cases of OSA in adults, and may represent a viable alternative to CPAP and MAD therapy alone. However, further studies are needed using 3D data to quantify the dentofacial advantages [27] of combined maxillo-mandibular oral appliance therapy.

\section{Acknowledgements}

This work was presented as a poster presentation at the American Academy of Dental Sleep Medicine in June, 2015. We would like to thank Dr Kim Cress, MD for her contributions to this work.

\section{Ethical Statement}

This study has been performed in accordance with the ethical standards laid down in the 1964 Declaration of Helsinki and its later amendments. All persons gave their informed consent prior to their inclusion in this study, including additional informed consent from all individual participants for whom identifying information is included in this article.

\section{Conflict of Interest}

Professor G. Dave Singh DMD PhD DDSc is the former CEO of BioModeling Solutions, Inc., a whollyowned subsidiary of Vivos BioTechnologies, Inc.

\section{References}

1. Calik MW (2016) Treatments for Obstructive Sleep Apnea. J Clin Outcomes Manag 23: 181-192.

2. Singh GD, Cress SE, McGuire MK, Chandrashekhar R (2012) Case report: Effect of mandibular tori removal on obstructive sleep apnea parameters. Dialogue 1: 22-24.

3. Deacon NL, Jen R, Li Y, Malhotra A (2016) Treatment of Obstructive Sleep Apnea. Prospects for Personalized Combined Modality Therapy. Ann Am Thorac Soc 13: 101118.
4. MacKay SG, Chan L (2016) Surgical Approaches to Obstructive Sleep Apnea. Sleep Med Clin 11: 331-341.

5. Başal Y, Akyıldız UO, Eryilmaz A (2015) Combined surgical treatment for severe sleep apnoea, to improve BiPAP compliance. BMJ Case Rep.

6. Azbay S, Bostanci A, Aysun Y, Turhan M (2016) The influence of multilevel upper airway surgery on CPAP tolerance in non-responders to obstructive sleep apnea surgery. Eur Arch Otorhinolaryngol 273: 2813-2818.

7. Wang J, Ma W, Xie Y, Hui P, Zhao L, et al. (2015) The curative effect analysis of continuous positive airway pressure combined with modified oral appliance in the treatment of severe OSAHS. Lin Chung Er Bi Yan Hou Tou Jing Wai Ke Za Zhi 29: 2044-2047.

8. Dioguardi A, Al-Halawani M (2016) Oral appliances in obstructive sleep apnea. Otolaryngol Clin North Am 49: 1343-1357.

9. Singh GD, Heit T, Preble D, Chandrashekhar R (2016) Changes in 3D nasal cavityvolume after biomimetic oral appliance therapy in adults. Cranio 34: 6-12.

10. Singh GD, Heit T, Preble D (2014) Changes in 3D midfacial parameters after biomimetic oral appliance therapy in adults. The Journal of Indian Orthodontic Society 48: 100104.

11. Morgan TD (2016) Novel approaches to the management of sleep disordered breathing. Sleep Med Clin 11: 173-187.

12. Singh GD, Olmos S (2007) Use of a sibilant phoneme registration protocol to prevent upper airway collapse in patients with TMD. Sleep Breath 11: 209-216.

13. Liao F, Singh GD (2015) Resolution of Sleep Bruxism using Biomimetic Oral Appliance Therapy: A Case Report. J Sleep Disord Ther 4: 204.

14. Singh GD, Griffin TM, Chandrashekhar R (2014) Biomimetic oral appliance therapy in adults with mild to moderate obstructive sleep apnea. Austin J Sleep Disord 1: 1-5.

15. Singh GD, Cress SE (2013) Craniofacial enhancement using a biomimetic oral appliance. Dent Today 32: 92-94.

16. Singh GD, Callister JD (2013) Use of a maxillary oral appliance for the resolution of obstructive sleep apnea. Cranio 31: 171175.

17. Singh GD, Cress SE (2013) Case presentation: Effect of full mouth rehabilitation and oral appliance therapy on obstructive sleep apnea. Dialogue 2: 18-20.

18. Singh GD, Wendling S, Chandrashekhar R (2011) Midfacial development in adult obstructive sleep apnea. Dent Today 30: $124-127$

19. Proffit WR, Frazier-Bowers SA (2009) Mechanism and control of tooth eruption: overview and clinical implications. Orthod Craniofac Res 12: 59-66.

20. Proffit WR, Fields HW, Sarver DM (2006) Contemporary Orthodontics. ( $4^{\text {th }}$ edn), Elsevier, Philadelphia.

21. Ottawa (ON): Canadian Agency for Drugs and Technologies in Health; 2014 Dec. CADTH Rapid Response Reports. Continuous Positive Airway Pressure Compared with Oral Devices or Lifestyle Changes for the Treatment of Obstructive Sleep Apnea: A Review of the Clinical and Cost-effectiveness.

22. Dieltjens M, Vroegop AV, Verbruggen AE, Wouters K, Willemen $\mathrm{M}$, et al. (2015) A promising concept of combination therapy for positional obstructive sleepapnea. Sleep Breath 19: 637-644. 
23. Chirinos JA, Gurubhagavatula I, Teff K, Rader DJ, Wadden TA, et al. (2014) CPAP, weight loss, or both for obstructive sleep apnea. The New England Journal of Medicine 370: 2265-2275.

24. Naughton MT, Monteith BD, Manton DJ, Dever P, Schachter LM, et al. (2015) Shorter Mandibular Length is Associated with a Greater Fall in AHI with Weight Loss. J Clin Sleep Med 11: 451-456.

25. Zhang XM, Tham CJ, Yin YL, Sun YQ, Zhou X (2015) A novel palatal implant surgery combined with uvulopalato- pharyngoplasty and inferior turbinate radiofrequency for the treatment of moderate to severe obstructive sleep apnea: a pilot study. Eur Arch Otorhinolaryngol 272: 1195-1202.

26. Uslu-Akcam O, Gokalp H (2015) Tongue Size and Posterior Airway Space Changes in Class III Malocclusion After Maxillomandibular Surgery: A Retrospective Study. Int J Orthod Milwaukee 26: 37-43.

27. Singh GD, Kim KY (2016) Facial enhancement using biomimetic oral appliance therapy in adults. Dental, Oral Craniofacial Research 2: 313-317. 